\title{
CONSIDERACIONES SOBRE EL CRETACICO DE LA CUENCA SUBANDINA DE BOLIVIA
}

\author{
FELIX GELSO REYES*
}

RESUMO O presente trabalho refere-se à estratigrafia dos sedimentos cretáceos da Bacia Subandina da Bolívia. $\mathrm{O}$ autor propõe muitas denominações para novas unidades estratigráficas reconhecidas no Grupo Tacuru e estabelece com maior precisão os limites e correlaçð̃es das unidades estratigráficas de superfície e subsuperficie. Também procura correlacionar as unidades litoestratigráficas da Bacia Subandina da Bolivia com as do Peru Oriental e da Bacia Andina da Argentina, Bolívia e Peru.

ABSTRACT The present paper is concerned with the stratigraphy of the Cretaceous sediments of the Subandean Basin of Bolivia. In fact, it can be considered a synthesis of the stratigraphy of the Gretaceous of this basin.

The author took the liberty of naming many new stratigraphic units recognized in the Tacuru Group, as well as establishing more precisely the limits and correlation of the stratigraphic units in the surface with those known in the subsurface.

The author also tried to correlate the lithostratigraphic units of the Subandean Basin of Bolivia with those of Eastern Peru and the Andean Basin of Argentina, Bolivia and Peru.

INTRODUCCION La Cuenca Subandina Boliviana-Peruana fue intensamente estudiada por numerosas compañías petroleras estatales y privadas, por el potencial petrolífero que la misma ofrecía; sin embargo, son relativamente pocas las publicaciones realizadas sobre tan vasta área debido a que el gran volúmen de informes geológicos existentes ha quedado en los archivos de las mismas compañias, existiendo sólo la esperanza de que un pequeño porcentaje de los mismos puedan llegar a conocerse en el futuro y ésto solamente en forma fragmentaria.

En lo que respecta al territorio de Bolivia dichos estudios fueron llevados a cabo, principalmente entre varias otras compañías, por Yacimientos Petrolíferos Fiscales Bolivianos, Standard Oil of Bolivia, Bolivian Gulf Oil, Shell Prospecting Ltd., Bolivia California Petroleum y la Chaco Petroleum.

Con referencia al conocimiento de los sedimentos cretácicos de tan extensa cuenca, ha constituído sin duda un real aporte la información suministrada por las numerosas perforaciones realizadas principalmente por Yacimientos Petrolíferos Fiscales Bolivianos y Bolivian Gulf Oil Co., sin cuya información parcialmente publicada hubiera sido francamente imposible llegar a establecer la correlación de síntesis que se ofrece en el presente trabajo.

Entre los trabajos publicados, que consignan cierta información sobre las sedimentitas cretácicas de la Guenca Subandina en relación con el presente trabajo, merece citarse siguiendo un órden cronológico los siguientes:

Heald y Mather (1922), cuando se hallaban al servicio de Richmond Levering Co., en el informe correspondiente, introducen en el léxico estratigráfico el término "Cajones limestone" con límites verticales diferentes a los utilizados en el presente trabajo bajo la denominación de "Formación Cajones" (Fig. 2).

*Universidad Nacional de Salta 
Mather (1922) introduce el término de "Formación Tacurú" para aquellas sedimentitas comprendidas entre la "Caliza de Vitiacua" y la "Formación Tatarenda", o entre su "Bermejo series" y la "Formación Tatarenda" en los lugares dondes se halla ausente la Caliza de Vitiacua; esto según lo puntualizado por su autor.

Padula (en Mauri, Padula y Aldazosa, 1956) de YPFB, da al término "Tacurú" el rango implícito de "Grupo" y consigna dentro del mismo los sedimentos comprendidos entre el tope de la "Caliza de Vitiacua" o de sus "Margas Multicolores" y el "Miembro Petaca", usando como sinónimo de "Areniscas Superiores" cuando llega a faltar el "Manto de Basalto".

Padula y Reyes (1958), dada la ambigüedad existente a esa fecha, sobre la denominación de "Tacurú" recomiendan concretar esta denominación para aquellos sedimentos comprendidos entre la discordancia que los separa de los Estratos del Chaco Inferior, por el tope, y la discordancia que los separa del "Basalto de Entre Ríos", Formación Ipaguazú, Caliza de Vitiacua o sedimentos de formaciones inferiores, en su base.

En este mismo trabajo se da a conocer varias otras denominaciones usadas para sedimentos cretácicos por diferentes geólogos de Standard Oil Co. y de YPFB, tratando de establecer sus posibles relaciones estratigráficas como su distribución. En este sentido se mencionan los términos de "Areniscas de Beu" de Schlagintweit (1939) y "Wall sands" tone" de Harrington (1926).

Díaz (1959) utiliza el término de "Formación Bala" para las Areniscas Bala de Schlagintweit (1939), pero no en la posición estratigráfica utilizada por este autor, sino para la parte alta del Beu que el presente autor la paraleliza con el "Miembro Arenisca Izozog" de la Formación Surutú (Fig. 3). Las "Areniscas de Bala" de Schlagintweit (1939) son sin duda terciarias y forman la parte basal del Grupo Beni.

Canedo Reyes (1960). En realidad esta es la fecha de publicación de un informe realizado en 1943. Este autor introduce el término de "Formación Eslabón" para la unidad litoestratigráfica que fue dada a conocer posteriormente por Perry (1963), como Formación Flora (Fig. 3).

Lamb y Truitt (1963 a y b) dan a conocer la introducción acertada del término "Formación Surutú" para el área de Santa Cruz realizada por los geólogos Chamot y Perry, con tres miembros, superior, medio e inferior.

Schlatter y Nederlof (1966) puntualizan ciertas correlaciones de la Formación Petaca, Formación Eslabón, Formación Tacurú y Formación Portón que necesariamente serán analizadas más adelante.

MARCO GEOTECTONICO DE LA SEDIMENTACION GRETACICA Con el nombre de Cuenca Subandina Boliviana-Peruana el autor designa a la cuenca originada por aquellos movimientos que mencionara Cecioni (1961) para la parte andiná del Norte de Chile y Perú, afectando los Andes Occidentales, pero que se hicieron extensivos también al área de la Cordillera Oriental y al ambiente de las Sierras Subandinas y Llanuras Chaco-. -Beniana.

En el área de Bolivia, Argentina y Perú, los siguientes elementos geotectónicos positivos delimitan esta cuenca durante la sedimentación cretácica: Dorsal Michicola, Arco Pampeano, Dorsal Izozog, Arco Subchiquitano, Escudo Brasílico y Dorsal Marañón (Fig. 1).

Dorsal Michicola Este elemento geotectónico tuvo al parecer una importancia fundamental durante la sedimentación del Cretácico, mostrando al Sur del paralelo $21^{\circ} \mathrm{S}$ una marcada inestabilidad con acusado grado de positividad y subdeformabilidad hasta defor- 


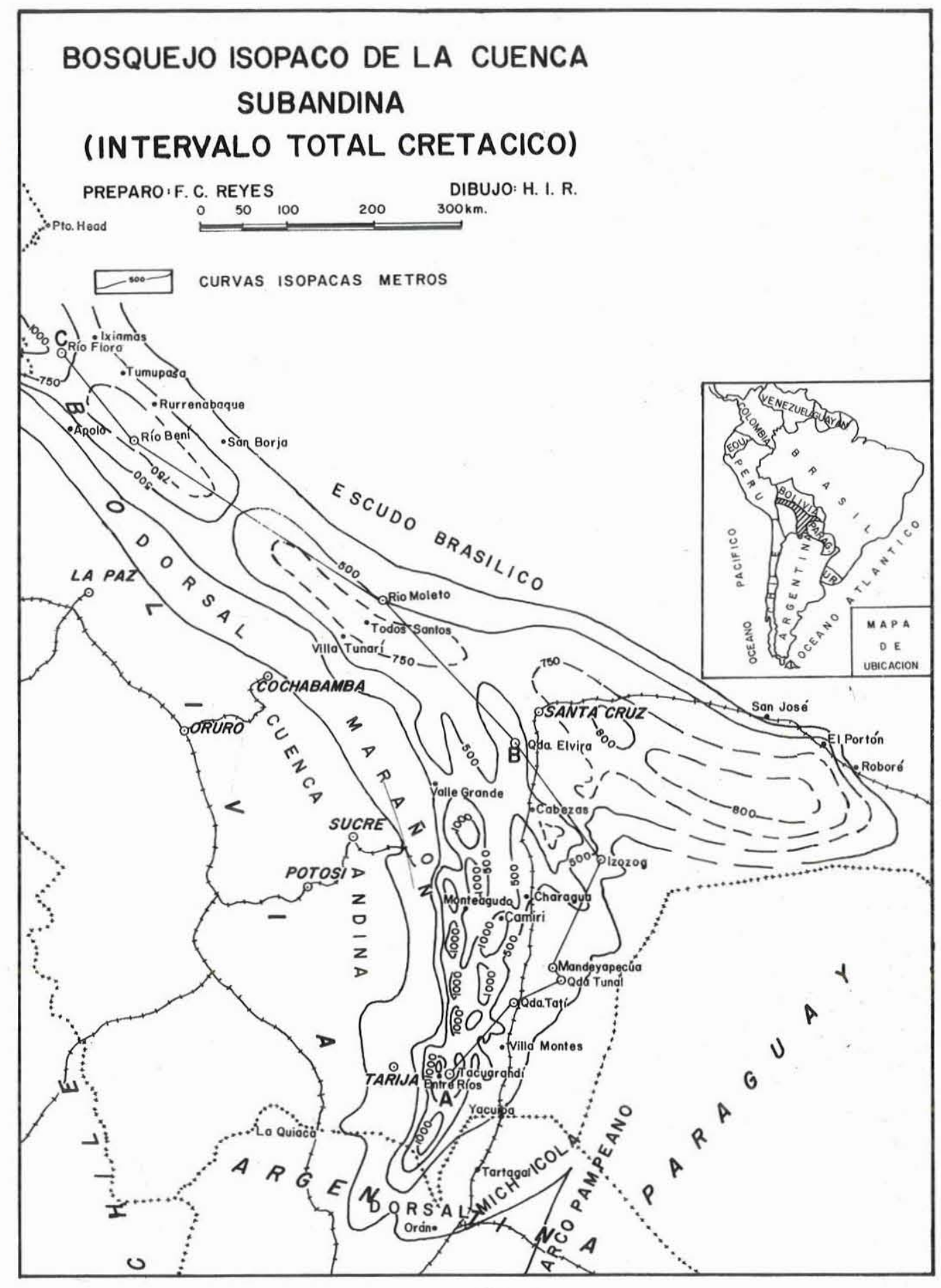

FIG. I 
mabilidad. Estos calificativos sobre el comportamiento de este elemento del cuadro geotectónico, así como de los otros a describir, están basados en el análisis del grado de tectonismo desplegado por el mismo durante el depósito del Grupo Tacurú (Cretácico), traducido tanto en su estabilidad vertical como en la tendencia positiva o subpositiva o en el grado de deformación sufrida durante el último diastrofismo del Terciario.

Esta "dorsal", tiene un rumbo NE a NNE. En su extremo occidental, área de Santa Victoria-Iruya, se confunde con la "Dorsal de Marañón" y al igual que esta última separa las dos grandes cuencas de la sedimentación cretácica, la "Cuenca Andina" en el Sur y Oeste, de la "Subandina Boliviana-Peruana" en el Norte y Este. En su prolongación Nororiental, en dirección al Paraguay, parece confundirse con el "Arco Pampeano", disminuyendo su movilidad y grado de positividad (Fig. 1).

Arco Pampeano Este arco parece corresponder o formar parte de aquel mencionado por Padula y Mingramm (1968, p. 328-329) con igual denominación. Su rama occidental se caracteriza por haber constituído un elemento subestable, positivo y subdeformable.

Dorsal Izozog Esta dorsal no ha sido individualizada en la Fig. 1, pero la misma fue definida en base a numerosos datos de subsuelo dados por perforaciones exploratorias e información geofísica en el área homónima. Su rumbo es parecido al del "Arco Pampeano" con el cual parece confundirse en dirección Este, para formar el Arco Subchiquitano, en el límite Paraguay-Bolivia siguiendo, aproximadamente, el paralelo $20^{\circ}$ de Latitud Sur. En dirección Oeste parece coincidir con el rumbo del Río Parapetí. Esta "dorsal" ha sido, al parecer, la que más ha contribuído, dentro de su carácter de positiva a subpositiva y subestable, a marcar las diferencias observadas durante la secuencia cretácica que se observa en las dos subcuencas que ella separa: la de Entre Ríos-Mandeyapecua, en la parte meridional, y aquella de Santa Cruz, en el Norte.

Escudo Brasílico Este Escudo aflorante en la parte Noroeste de la Fig. 1 se ha comportado como un elemento estable, positivo e indeformable. Entre el borde aflorante de este Escudo y el Arco Subchiquitano, queda confinada una subcuenca que el autor la denomina "Subcuenca Roborê" prolongación oriental de esa otra ya mencionada como Subcuenca Santa Cruz.

Existen otras dorsales menores que han subdividido la Cuenca Subandina Boliviana-Peruana al NW de la ciudad de Santa Cruz, entre las que se puede mencionar la "Dorsal Ichilo" que separa la Subcuenca de Santa Cruz de esas otras del Noroeste denominadas en conjunto "Subcuenca Beniana" (Fig. 1).

El bosquejo isopáquico de la Fig. 1, que representa en la Cuenca Subandina la totalidad de la secuencia cretácica, no llega a esbozar claramente las dos subcuencas, de Santa Cruz y Entre Ríos-Mandeyapecua, por cuanto si bien en la primera de estas o sea en la más septentrional se hallan representadas todas las unidades litoestratigráficas del Grupo Tacurú, en la meridional faltan las unidades inferiores, pero adquiere mayor espesor la unidad superior (Formación Bororigua) que llega a neutralizar las diferencias observadas en los isopáquicos de las unidades basales.

ESTRATIGRAFIA DEL CRETACICO DE LA CUENCA SUBANDINA Grupo Tacurú Toda la secuencia cretácica de la Cuenca Subandina Boliviana se halla comprendida dentro de lo que se conoce con el nombre de Tacurú, nombre éste dado por Mather (1922, p. 741-746) en el rango de formación, el cual fue redefinido por Padula y Reyes (1958, p. 50-51) para aquellos sedimentos que están comprendidos entre la dis- 
cordancia que los separa de la base de los Estratos del Chaco Inferior, por el tope, y la discordancia que los separa del Basalto de Entre Ríos, Formación Ipaguazú, Caliza Vitiacua o sedimentos de formaciones inferiores en su base (Fig. 2).

Las sedimentitas del Tacurú han planteado en todo tiempo problemas estratigráficos de difícil solución, suscitando diversos tipos de controversias, no sólo con respecto a sus límites superior e inferior o a su correlación lateral, sino hasta en lo que se refiere a su propia identificación en ciertos sectores de la cuenca donde ellos fueron depositados.

Al autor le cupo realizar junto con el Ing. J. Oblitas de Y.P.F. Bolivianos (1968) la correlación, dentro del marco de la zona Subandina, de las diferentes unidades observables tanto en superficie como en el subsuelo, que completaron las ya llevadas a cabo por el autor (1963). Los resultados de estos trabajos condujeron a sugerir una nomenclatura a fin de facilitar los estudios de litofacies y de valoración de áreas petroleras, por la importancia adquirida por estas sedimentitas, en el área de Santa Cruz, como productoras de hidrocarburos.

El autor, en el presente estudio, ha salido del marco de la propia cuenca Subandina de Bolivia y ha tratado de correlacionar las formaciones del Grupo Tacurú con aquellas cretácicas depositadas en la Cuenca Andina, vinculándolas, asimismo, con las aflorantes en el Oriente Peruano.

A fin de aclarar la discusión sobre la correlación estratigráfica en la Cuenca Subandina Boliviana-Peruana se cree conveniente dar a continuación un resúmen de las características litológicas y definición de las unidades litoestratigráficas utilizadas en el presente trabajo, basadas principalmente en el estudio de Reyes y Oblitas (1968). Estos autores, al reconocer dentro de las sedimentitas cretácicas diferentes unidades formacionales, han elevado a categoría de grupo la formación Tacurú de Mather y, dentro de esta unidad mayor, han reconocido tres unidades formacionales que, de abajo hacia arriba, ellas son: 1) Formación Surutú, 2) Formación Cajones y 3) Formación Bororigua. Sólo la Formación Surutú fue subdividida en tres miembros, que siguiendo el mismo órden ellos fueron denominados: Peña Blanca, Elvira y Arenisca Izozog.

Formación Surutú Con este nombre, geólogos de la Bolivian Gulf Oil Co., Lamb y Truitt (1963 a y b) designaron a una "secuencia de tres miembros compuestos por areniscas amarillentas y rojizas friables de $500 \mathrm{~m}$ de espesor. El miembro inferior está principalmente compuesto por areniscas cuarzosas amarillas, bien seleccionadas, de estratificación delgada a masiva. El miembro mediano se caracteriza por ser entrecruzado y de color generalmente rojizo. El miembro superior es generalmente amarillento, calcáreo, con capas ocasionales de areniscas duras silíceas y calcáreas. Este se vuelve progresivamente más calcáreo hacia su parte superior. La sección tipo de la Formación Surutú se éncuentra en la Quebrada Elvira y la co-tipo en el Río Alto Surutú" (1963 b, p. 19).

Si bien la delimitación de los diferentes miembros de la Formación Surutú, en la localidad tipo, corresponde a los que el autor denominara junto con Oblitas (1968), con los nombres expuestos más arriba, Lamb y Truitt no identificaron el miembro inferior en sus secciones de los Pozos de Caranda y confundieron en el Pozo Río Grande 1 el Miembro Medio Elvira con la Formación San Telmo de edad Carbonífera y la parte expuesta del Miembro Inferior la incluyeron dentro de la Formación Escarpment (1963, p. 29, Fig. 3). Vide Fig. 2.

Esta Formación Surutú, Lamb y Truitt la paralelizaron con el Tacurú de la zona meridional, lo que ya concordaba en parte con la idea del autor (1963) de acuerdo a los límites y nomenclatura graficada en la Fig. 2. 


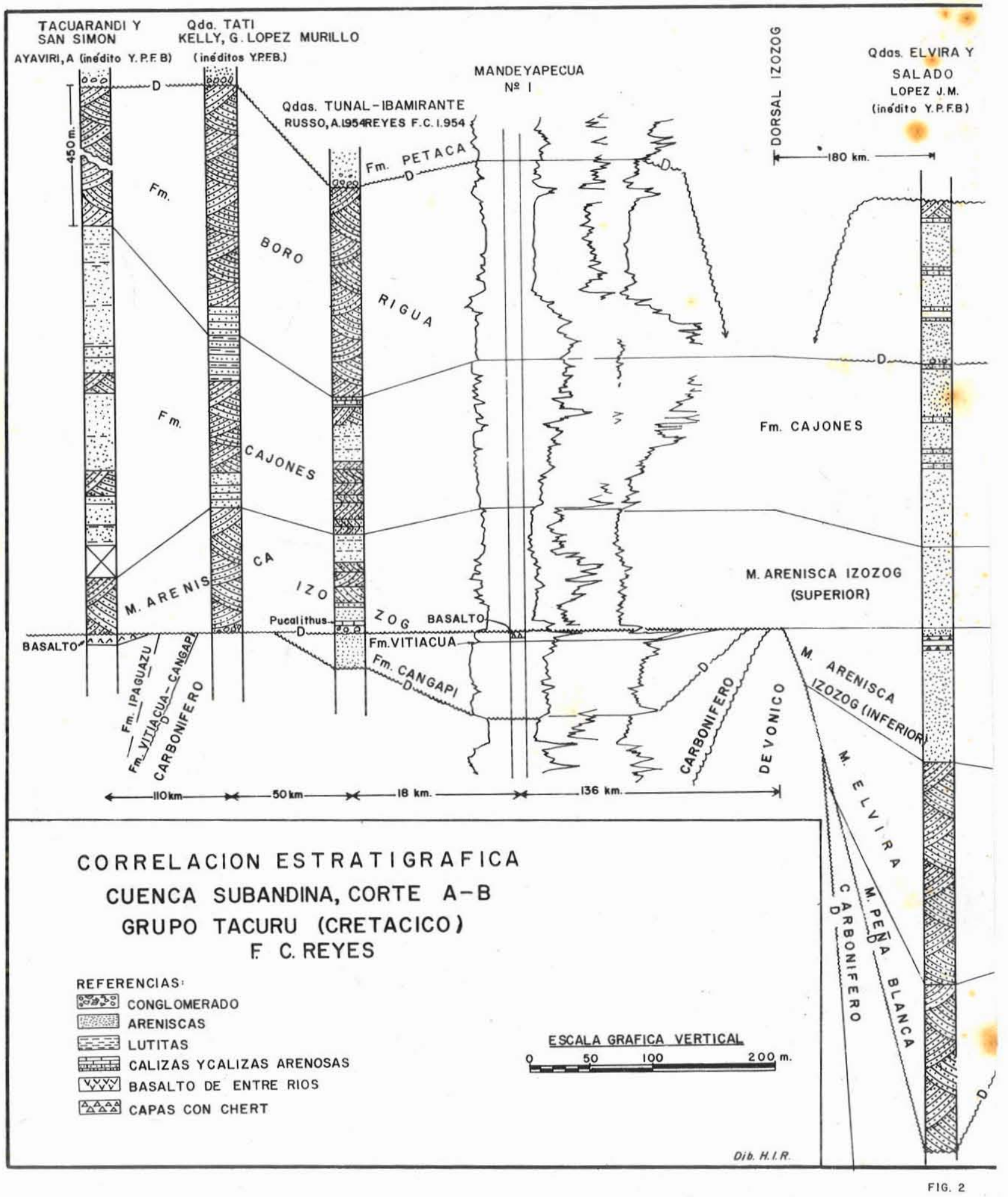


PERFIL TIPO, (INO-ELECT)

(CUENCA SANTA CRUZ)
REYES, F. C. HEAL.D, K.C.

OBLITAS, J. MATHER, K.F.

$1.969 \quad 1.922$
LAMB, W. C

TRUIT, $P$. $B$

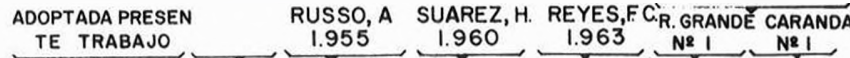

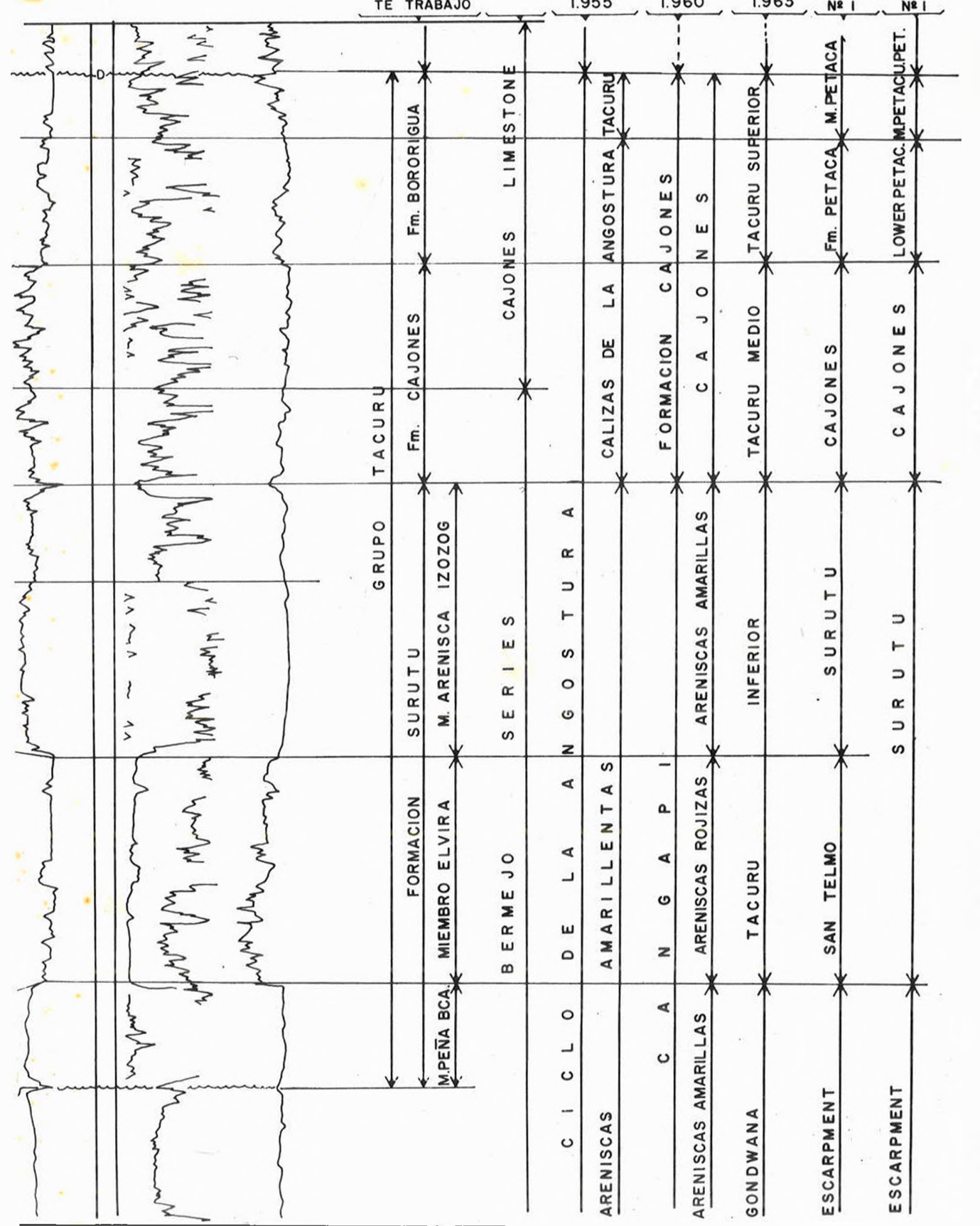


Miembro Peña Blanca de la Formación Surutú Reyes y Oblitas (1968) denominaron con este nombre al miembro inferior de la Formación Surutú de Lamb y Truitt (1963), eligiendo como sección tipo el camino al Pozo Camiri n. ${ }^{\circ} 1$, vecino a la localidad del mismo nombre en el área del sector del paraje conocido como Peña Blanca, que tipifica el color dominante de esta unidad.

Este miembro, en la sección tipo, fue correlacionado en años anteriores, en varios de los informes de S.O. Co. of Bolivia y en trabajos propios de Y.P.F. Bolivianos, como parte de la Formación Cangapi, y así lo hicieron Padula y Reyes (1958, p. 41) que la vincularon más propiamente con las "Areniscas Calcáreas" existentes debajo del Vitiacua.

En el área de Camiri, esta unidad está formada de abajo hacia arriba de:

a) Arcilitas marrón rojo-oscuro, con diaclasas rellenadas de carbonato de calcio, con varias intercalaciones de areniscas blanquecinas y láminas de areniscas grano fino, de color rojo pardusco.

b) Areniscas blanquecinas grano fino, en finas capas con intercalaciones rojas, verdes y amarillas; friables.

c) Areniscas gris parduscas y rojo pardusco grano fino a mediano, laminadas con entrecruzamiento; friables.

d) Areniscas gris blanquecinas de grano grueso, muy duras, calcáreas, en bancos hasta $0,60 \mathrm{~m}$ de espesor, alternando con arcilitas calcáreas, verdes y rojas.

e) La parte del tope de este miembro está formada por areniscas gris blanquecinas grano fino, laminadas, algo deleznables y areniscas gris blanquecinas de grano muy fino calcáreas, duras y compactas de $0,70 \mathrm{~m}$ de espesor. Presentan concreciones calcáreas, duras.

El espesor de este miembro en el área de Camiri, Quebradas Evangelista y Justiniano es de unos 350 metros.

Si bien este miembro en la Subcuenca de Santa Cruz en varias secciones de los pozos perforados no ha sido observado, en la Subcuenca de Entre Ríos-Mandeyapecua, no fue depositado, como tampoco lo fue al Noroeste de Santa Cruz en la Subcuenca Beniana, entre el Río Ichilo y la frontera Bolivia-Perú (Fig. 2). En la Subcuenca de Roboré, en el área de Portón, su espesor es del orden de $125 \mathrm{~m}$.

Miembro Elvira de la Formación Surutú La sección tipo de este miembro es tanto la que eligiera Lamb y Truitt (1963 a y b) para su Formación Surutú, es decir el curso de agua de nombre Elvira localizado en el flanco oriental de la Serranía de Espejos, como la quebrada Salado vecina a la misma. En la sección tipo este miembro alcanza una potencia de $180 \mathrm{~m}$ (Fig. 2) y se halla formado por areniscas de colores rojizos de grano medio a fino, limosas, bien redondeadas, presentando reacción calcárea y fuerte entrecruzamiento.

Esta unidad estratigráfica se la reconoce bien en la Subcuenca de Santa Cruz o sea aproximadamente al Norte del Río Parapetí, no habiendo sido observada al Sur de la Dorsal de Izozog. Al Noroeste de Santa Cruz de la Sierra, en la Subcuenca Beniana (Figs. 1 y 3), el Miembro Elvira es la unidad estratigráfica que forma la mayor parte de la secuencia cretácica expuesta en esa zona y que se la reconoce, en esa área como "Formación Beu’’ designada así por Schlagintweit (1939).

Miembro Arenisca Izozog El miembro superior de la Formación Surutú fue denominado por Reyes y Oblitas (1968) como Arenisca Izozog, tomando tal nombre de una unidad del subsuelo reconocida por geólogos de Y.P.F. Bolivianos en la región del mismo nombre, al Este de la localidad de Camiri, sobre el borde septentrional de la Dorsal de Izozog, 
zona donde forma la base de la secuencia cretácica del Grupo Tacurú. Sin embargo, la unidad que se describe en el presente trabajo como Arenisca Izozog no corresponde sólo a la parte basal de aquella unidad reconocida en el subsuelo, sino se halla integrada también con una parte superior de sedimentitas vinculadas a tal unidad y así se lo reconoce en las exposiciones de superficie (Fig. 2).

La sección tipo para esta unidad es la misma que para el Miembro Elvira.

El Miembro Arenisca Izozog está caracterizado por areniscas amarillas de reacción calcárea, muy peculiares en la Subcuenca de Santa Cruz como en la Subcuenca Beniana. Dentro de este paquete arenoso, en la parte superior del mismo, se han observado hasta dos niveles de chert (ftanita) situados más bien cerca del tope de la Arenisca Izozog (Inferior). Se ha visto que estos niveles no ofrecen una continuidad regional, sino por el contrario tienen carácter lenticular y lo normal es encontrar que se halle representado sólo uno de ellos. Estos niveles de sílice fueron paralelizados por geólogos de las compañías Shell y California, en los pozos de Monte Verde y Monte Cristo, con el denominado por ellos "Horizonte guía Petaca" en el área de Santa Cruz y "Horizonte guía Eslabón" en el Noroeste (Schlatter y Nederlof, 1966, p. 31), considerándolos como "una superficie silicificada característica de un período de no deposición en un clima árido" y vinculándolos con el Cajones, que como se sabe lo suprayace.

Al Sur de la Dorsal de Izozog, en la Subcuenca de Entre Ríos-Mandeyapecua, sólo se hallan representadas la parte superior de la Arenisca Izozog de Reyes y Oblitas (1968), como acontece por ejemplo en la Quebrada de Ibamiranti, del área de Mandeyapecua, donde se observa cierto cambio de color en las areniscas. En esta sección, esta parte alta del Miembro Arenisca Izozog descanza mediante un conglomerado basal, sobre sedimentitas de la Formación Cangapi. En las intercalaciones calcáreas que presenta, consistentes en tres o cuatro banquitos de calizas grises, de tres a cinco centímetros de espesor cada uno, el Dr. Russo junto con el autor encontraron los primeros Pucalithus de Steinmann o Fósil Problemático de Bonarelli, que se tenga mención, en la Cuenca Subandina Boliviana, dentro de los sedimentos asignados al Tacurú, mencionados en Padula y Reyes (1958).

Con posterioridad el autor da a conocer (1962, p. 795) que, en la Sierra de Charagua, geólogos de la Chaco Petroleum y Gulf Oil Corp. encontraron, igualmente, estos Pucalithus en capas hoy consideradas por el autor como pertenecientes a las Areniscas Izozog, las cuales fueron correlacionadas erróneamente, por los citados geólogos, con la Formación Ipaguazú de edad Triásica.

A esta unidad litoestratigráfica pertenece también, sin duda y en parte, los encontrados por Oblitas (1962), en el área de Bororigua, al Sur de Camiri; aunque por la descripción que da este colega las varias muestras de Pucalithus observadas por este geólogo parecen estar localizadas más abundantemente en la Formación Cajones suprayacente.

En los pozos Carandaiti 1, 2 y 3 en niveles que fueron considerados erróneamente como pertenecientes a la Formación San Telmo de edad Carbonífera (Reyes 1962, p. 793; y Lamb y Truitt 1963 b, p. 18) y que ahora se los paraleliza con la parte alta del Miembro "Arenisca Izozog", se encontraron algunos fósiles considerados por el Dr. Paul Tasch, 1960 (Informe inédito a Gulf Oil Corporation), como Estéridos, probablemente Isaura de edad pérmica. Sin embargo, otro lote similar de los mismos niveles, enviado a Shell Prospecting Ldt. y estudiado en Europa, los especialistas arribaron a resultados contradictorios sin descartar la posibilidad de que se tratasen de formas cretácicas. Por estas razones se piensa que los fósiles no son diagnósticos.

Un poco más al Norte de la Quebrada de Ibamiranti, en la Serranía de Mandeyapecua, la parte "alta" de la Arenisca Izozog se apoya discordantemente sobre una superficie de 
colada basáltica, el asi denominado por Padula y Reyes (1958) Basalto de Entre Ríos. Esta misma relación se observa en la zona de Entre Ríos, sección Tacuarandí (Río Salado) y San Simón (Fig. 2), aunque quizás sean términos más altos del mismo miembro los que se apoyan sobre la colada de basalto. Existe, sin embargo, una incógnita y ella se halla relacionada con el hecho de que muy posiblemente parte de la Formación Ipaguazú, que infrayace al "basalto", en el área de la localidad de Entre Ríos, pertenezca en realidad al ciclo del Cretácico y no al del Triásico, por cuanto la gran uniformidad en sentido areal observada en el techo de la Formación Ipaguazú, denominada informalmente por geólogos de Y.P.F. Bolivianos "areniscas té con leche" de poco espesor, no nos habla en favor del gran hiatus existente entre Triásico y Cretácico Superior, tiempo en el que necesariamente debió estar sujeta a cierto tipo de erosión. De ser esto correcto el límite Triásico* -Cretácico, se lo debiera buscar en la interpretación de la remoción de ciertas capas de yeso que forman un tipo especial de conglomerados, aunque todo esto a solo título de hipótesis.

En el área Noroeste de Santa Cruz de la Sierra, Subcuenca Beniana, las sedimentitas paralelizables con el Miembro Arenisca Izozog, integrando la parte "alta" de la Formación Beu, se hallam expuestas con las siguientes características generales: areniscas amarillentas y cremas, estratificadas en bancos gruesos y masivos, duros debido a la silicificación y que forman crestas bien definidas en la topografia, infrayaciendo discordantemente por debajo de la Formación Eslabón de Canedo Reyes (1960) o Formación Flora de Perry (1963) (Fig. 3).

En el área de Camiri, el techo del Miembro Arenisca Izozog está formado por areniscas rosa pálido, grano medio, dispuestas en finas capas regulares, bien silicificadas, compactas y duras, de 8 a $10 \mathrm{~m}$ de espesor, las que erróneamente fueron incluídas dentro de la Formación Petaca por algunos geólogos de la compañía petrolera S.O. Co. of Bolivia y sería paralelizable a lo que Schlatter y Nederlof (1966) denominan "Petaca Keybed" y lo paralelizan con Cajones de Lamb y Truitt (1963b p. 31). Sobre esta unidad en Camiri, se apoyan recién los conglomerados basales del Grupo Chaco (Conglomerado de Petaca). En la Subcuenca de Roboré, en el área de Portón, su espesor es del orden de los $90 \mathrm{~m}$ aproximados.

Formación Cajones La denominación de "Cajones" fue introducida en léxico estratigráfico de Bolivia por Heald y Mather (1922, p. 553-570) para su formación: "Cajones Limestone" del área de Santa Cruz de la Sierra, utilizando como localidad tipo la cadena frontal, denominada hoy de Terebinto, donde reconocio $240 \mathrm{~m}$ de sedimentitas para esta su unidad. De acuerdo con la Fig. 2, donde se ha graficado los límites correspondientes a unidades estratigráficas de algunas de las nomenclaturas utilizadas, se vé que Heald y Mather incluyeron dentro de sus Cajones Limestone aproximadamente la mitad superior de la Formación Cajones descrita en este trabajo, como asimismo la Formación Bororigua del presente estudio y la parte basal de la Formación Petaca.

Esta formación tiene como localidad tipo la misma asignada por Heald y Mather y más propiamente elegida debe corresponder al Río Alto Surutú.

Esta formación descansa en concordancia sobre la Formación Surutú, con un espesor, en el área tipo, de $130 \mathrm{~m}$. Se halla constituída por areniscas calcáreas y calcarenitas de colores blanquecino, gris blanquecino o ligeramente rosado, llevando concreciones calcáreas y silíceas. La estratificación es gruesa a masiva, en capas de 1 a $5 \mathrm{~m}$ de espesor, con algunos horizontes muy delgados de arcilitas. Es una unidad muy resistente a la erosión y muy peculiar y de fácil reconocimiento en el subsuelo por sus características eléctricas 


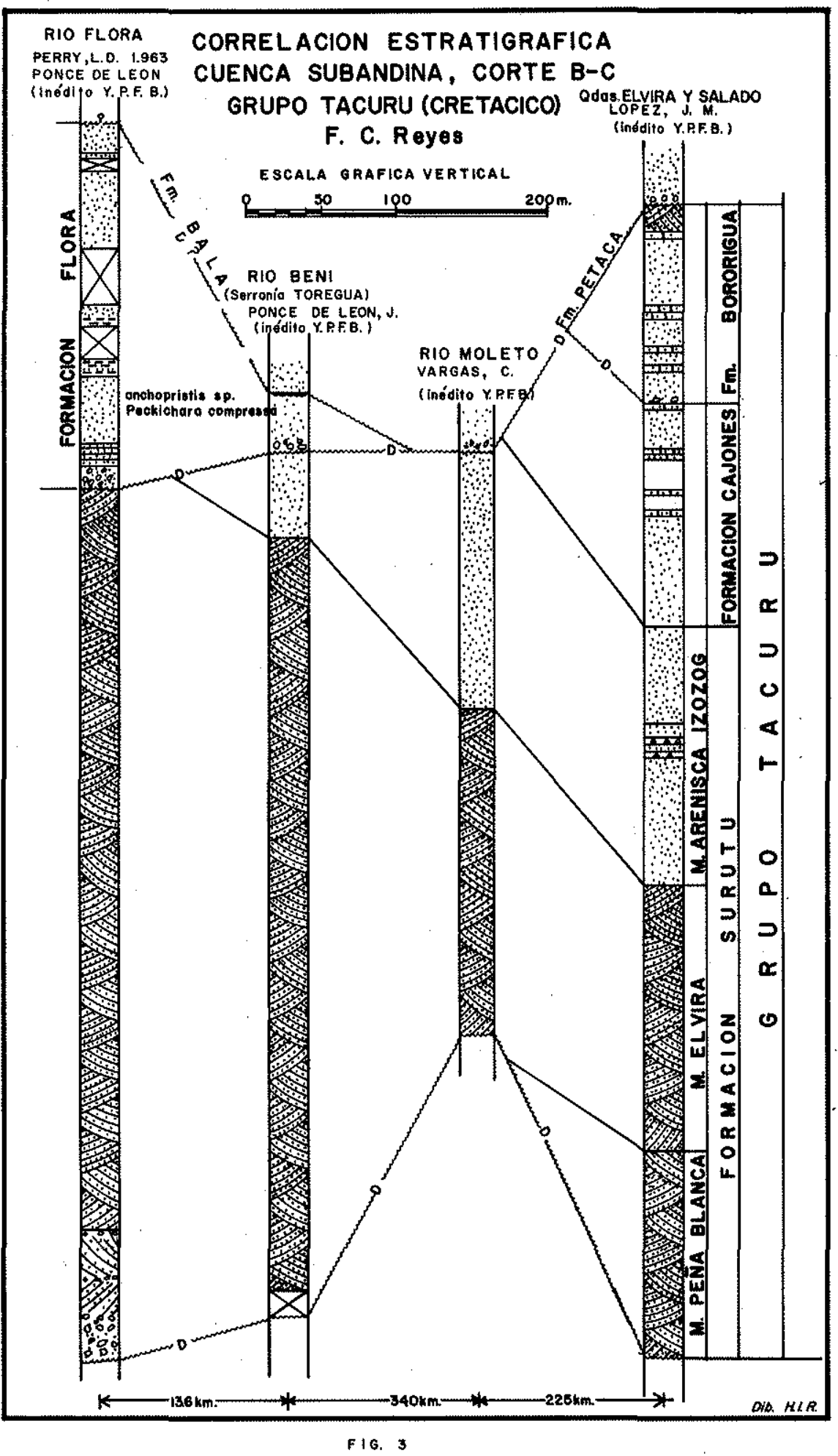


como puede apreciarse en la Fig. 2. Es una de las formaciones productoras de petrólı en la Subcuenca de Santa Cruz.

En la Subcuenca de Entre Ríos-Mandeyapecua, al Sur de la Dorsal de Izozog, se halla bien expuesta en la Serranía de Mandeyapecua, donde fue denominada por el Dr. A. Russo y el autor, (Russo, 1955 a) "Areniscas Blanquecinas" y son de reacción calcárea, pero con delgadas intercalaciones arcillosas lenticulares. El contenido calcáreo disminuye notablemente en la Subcuenca de Entre Ríos-Mandeyapecua con respecto a lo observado en la de Santa Cruz, pero ello no dificulta mayormente su correlación, especialmente en ciertas áreas donde localmente aumenta el contenido de este material (Oblitas, 1962).

El único resto fósil encontrado en esta formación fue un hueso de dinosaurio indeterminable, realizado por Ericson de la Compañía Bolivian Gulf Oil Corp. efectuado en la Serranía de Mandeyapecua.

Se debe aclarar que la misma Formación Bororigua en la Subcuenca de Santa Cruz lleva alto contenido calcáreo y en superficie, por ejemplo, en la Sierra de Terebinto y Espejos es muy difícil separarla de la Formación Cajones infrayacente como se lo hace en el subsuelo al disminuir el contenido de calcáreo, razón por la cual no existe una identidad litológica entre ambos tipos de unidades, de superficie y subsuelo. Sin embargo, esto es local, pues en dirección hacia el Sur y Noroeste la separación se hace más fácil y la vinculación de la unidad superior con la Formación Bororigua de la Subcuenca de Entre Ríos-Mandeyapecua se clarifica.

El techo de la Formación Cajones, según Lamb y Truitt (1963 b, p. 19), es discordante y marcado a veces por un conglomerado basal formado por material retrabajado de Cajones. Esto es verdad en lo que respecta a la existencia de un conglomerado en la base de lo que el presente autor considera la parte correlacionable con la Formación Bororigua de la zona de Entre Ríos, pero no lo es en la homologación de tal conglomerado con el correspondiente a la Formación Petaca, por cuanto, si bien fue difícil detectarlo, se logró sacar testigos en algunos pozos perforados por YPFB, como por ejemplo en uno de la estructura de Río Seco, donde se pudo ver el típico conglomerado de Petaca de los afloramientos de superficie descansando sobre la Formación Bororigua. Estas evidencias no hacen aceptable la correlación de Lamb y Truitt graficadas en la Fig. 2 con respecto al límite entre Terciario y Cretácico.

La Formación Cajones no ha sido reconocida en la Subcuenca Beniana. No se sabe si llegó a depositarse o bien si fue erosionada antes del depósito de la Formación Flora o de los mismos sedimentos terciarios.

En Camiri se hallan ausentes las sedimentitas correspondientes a esta formación, pero ello se debe, sin duda, a la erosión pre-Petaca.

En la Subcuenca de Roboré, área del Portón, sólo parece estar aflorandó la mitad inferior de la formación con más o menos $130 \mathrm{~m}$ de espesor.

Formación Bororigua. Esta denominación fue dada por Reyes y Oblitas (1968) para aquellas sedimentitas aflorantes en la Subcuenca meridional de Entre Ríos-Mandeyapecua, correspondientes a la parte más alta aflorante del Grupo Tacurá, constituídas por areniscas de colores rojizos y rojo-anaranjado, de grano predominantemente medio, arcillosas y laminadas con fuerte entrecruzamiento de tipo eólico, que llevan a veces delgados niveles conglomerádicos especialmente en su parte basal.

La sección tipo es la que eligiera Padula y Reyes (1958, p. 51) para todo el Tacurú, o sea las nacientes de los ríos Salado y Timboy, poco aguas abajo de las localidades homónimas y sobre el flanco occidental del Anticlinal del Río Salado o Anticlinal de Tacuarandí como se le suele denominar, también. La sección co-tipo es el área de la Estructura de 
Bororigua en la Serranía de Sararenda donde dicha serranía es cortada por el camino que une la localidad de Cuevo con la zona de Pirarenda, al SSW de Camiri.

En la Subcuenca de Santa Cruz, en el subsuelo de la misma, es fácil de distinguir en los registros eléctricos (Fig. 2), por su posición, su baja resistividad y su bajo potencial espontáneo, habiéndose observado en los recortes de terreno y testigos que en muchas secciones se halla formada preferentemente por limolitas y arcilitas, con mayor o menor contenido calcáreo pero con incremento del contenido arenoso en la parte inferior, donde en algunos pozos se ha encontrado un conglomerado considerado basal.

Esta unidad litoestratigráfica sería la única que se halla representando al Cretácico en la Estructura de Agua Salada, cerca de la frontera Bolivia-Argentina, descansando con sus $75 \mathrm{~m}$ por encima de capas de la Formación Vitiacua y por debajo de la típica Formación Petaca (Areniscas Superiores de Bonarelli, 1921).

Esta unidad estratigráfica es denominada "Formación Naranjillos" en el subsuelo del campo gasifero del mismo nombre, cerca de Santa Cruz.

Es una unidad que tiene mucho interés económico en vista de los varios campos que en sus niveles porosos y permeables, encontraron producción.

El techo de la Formación Bororigua se halla bien definido en toda la Cuenca Subandina y está caracterizado por la discordancia basal terciaria ubicada en la base de la Formación Petaca de edad eocena a oligocena, pues datos palinológicos de la Formación Yecua superpuesta parecen indicar para ésta una edad oligocena-miocena. Con la Formación Petaca comienza un nuevo ciclo de depósito, paralelizables a ese de las Areniscas Superiores al Norte de la Dorsal de Michicola. La base de la Formación Bororigua parece corresponder, en ciertas áreas, con una superficie de erosión o discordancia originadas por algún movimiento intercretácico, caracterizada por un conglomerado que el autor lo correlaciona con aquel encontrado por Perry (1960) en la Subcuenca Beniana, en el Río Flora, afluente del Río Madidi, en las cercanías de Ixiamas al NW de Rurrenabaque, en la base de su Formación Flora, equivalente lateral de la Formación Eslabón de Canedo Reyes (1960). Esto en virtud de que tal unidad Flora de $240 \mathrm{~m}$ de espesor se apoya discordantemente sobre la Formación Beu (Miembros Elvira y Arenisca Izozog de la Subcuenca de Santa Gruz) y por debajo de la Formación Bala de la cual se halla separada por una probable discordancia (Fig. 3). Sin embargo, esta relación que parece clara, algunos ge6logos de Y.P.F. Bolivianos que estudiaron esta zona se sintieron inclinados a considerar la Formación Flora o Eslabón como equivalentes laterales de la Formación Petaca, como lo consideró López Murillo (1967, p. 21). Sin embargo, no debe olvidarse la edad cretácica de la Formación Flora, en vista de la fauna y flora encontrada por Perry (1960), la que incluye (p. 1800 ) gastrópodos, ostrácodos y escamas de peces aún sin determinar, como asimismo, dientes de peces identificados en el American Museum of Natural History como Onchopristis sp. y varias especies de charófitas, incluyendo Peckichara compressa (Knówlten) que según comunicación epistolar de Raymond E. Peck, de la Universidad de Missouri, corresponden a una zona cerca del mismo tope del Cretácico. Onchopristis, en cambio, sólo indica edad cretácica. Perry correlaciona tentativamente su Formación Flora con la Formación Tacurú (?) de la Zona Subandina meridional. Los fósiles recogidos por Ponce de León, según comunicación verbal del Dr. H. López Murillo, habrían sido determinados en Francia como Gasteroclupea branisai, Pucapristis branisi y Pucalithus. De todas maneras estos fósiles son menos diagnósticos que Peckichara compressa para ubicar la sección dentro de la secuencia cretácica. Sin embargo, aportan mayor información sobre el ambiente de depósito de la Formación Flora que se considera parálico a mesoparálico.

Actividad Efusiva. Coincidiendo con un hiatus que abarcaría desde el Triásico Superior a la parte alta del Coniaciano (?), y regionalmente sobre diferentes unidades litoestratigrá- 
ficas del Grupo Cuevo (Triásico), se suele encontrar, dentro de cierta parción de la Subcuenca de Entre Ríos-Mandeyapecua, y muy especialmente en la parte occidental de la misma, coladas de basalto denominadas "Basalto de Entre Ríos" por Padula y Reyes (1958). De esta manera se lo observa en la vecindad de Entre Ríos, secciones de Tacuarandí y San Simón (Fig. 2), apoyarse sobre sedimentitas de la Formación Ipaguazú o sea la unidad superior del Grupo Cuevo. En cambio en la zona de Mandeyapecua (Fig. 2) y en la margen derecha del Río Parapetí, donde éste corta la Serranía de Carahuaichu al SE de Camiri, se lo encuentra sobre la Formación Vitiacua o unidad media del Grupo Guevo.

No hay mención de la existencia de efusiones basálticas en la Subcuenca de Santa Gruz fuera de aquellas existentes en el límite con la Subcuenca de Entre Ríos-Mandeyapecua, mencionada anteriormente sobre el Río Parapetí y que pasan a la margen izquierda del citado curso de agua. Ultimamente Ponce de León (Reyes y Oblitas, 1968) encontró un filón capa de basalto en la sección del Río Yanamayu vecina a la frontera Boliviana-Peruana al NW del Río Flora en capas de la Formación Beu paralelizables con el Miembro Elvira de la Formación Surutú (Fig. 3).

El Basalto de Entre Ríos que tiene espesores desde menos de $1 \mathrm{~m}$ hasta cerca de $90 \mathrm{~m}$ se presenta, por lo general con un color gris negruzco y en la parte central de su área de distribución, en los alrededores de Entre Ríos, pareciera apoyarse sobre el mismo nivel de la Formación Ipaguazú, en las así denominadas informalmente "areniscas té con leche" por geólogos de Y.P.F. Bolivianos. Esta situación hizo prever la posibilidad al autor de que se tratara de una efusión inter-Tacurú en esta área, es decir, que parte de lo que se considera como sedimentitas de la Formación Ipaguazú de edad triásica, en realidad podrían pertenecer al ciclo de deposición del Cretácico (Tacurú), como ya fue puntualizado anteriormente. De ser esto cierto, al menos en esta parte de la cuenca, daría mayor apoyo a la datación dada a la parte superior del Miembro Areniscas Izozog de la Formación Surutú (Fig. 4), como principalmente Santonense y a la parte inferior como de edad Coniaciana.

El autor fue informado por personeros de la Compañía Petrolera Bolivian Gulf Oil Corp., en años atrás, de varias determinaciones sobre edades absolutas de basaltos aflorantes en diversas partes dentro de la Subcuenca de Entre Ríos-Mandeyapecua llevadas a cabo en sus laboratorios de Pittsburg, USA, sin mencionar el método empleado. Los resultados arrojados por estas determinaciones son bastante dispares, máxime si se considera que ocupan la misma posición estratigráfica, aunque ellas correspondan a un posible gran hiatus que va desde el Triásico Superior hasta el Neo-Coniaciano(?). Algunas de estas contradictorias determinaciones son sobre basaltos aflorantes sobre los dos flancos de un mismo anticlinal. Los valores registrados oscilan desde una edad menor a los 63 millones de años hasta una sola que acusa 233 m.a. Hay algunas con valores de 104 a 108 m.a. pero esa del Sinclinal Tacuarandí (Río Salado), Fig. 1, acusa en su flanco oriental 82 m.a., pero en el occidental 71 m.a. Estas variaciones de edad observadas pueden deberse, en parte, a aspectos inherentes al método mismo utilizado, además de los problemas de obtención de muestras en zonas húmedas. No otra explicación puede tener el valor de - 233 m.a. que ubica la efusión en el Pérmico cuando por debajo de la misma se encuentra, en esa localidad, la Formación Vitiacua, de edad Noriense Superior.

De todas maneras el autor se siente inclinado a correlacionar las efusiones basálticas de la Subcuenca de Entre Ríos-Mandeyapecua con aquella observada en la Cuenca Andina de Bolivia o sea con la segunda generación de basaltos del área de Betanzos que dió una edad Coniaciana tardía (Kriz, Cherroni et al., 1966), y por lo tanto con los basaltos de la Subcuenca de Alemanía (Argentina). El filón capa de basalto de la sección del Río 
CUADRO DE CORRELACION CRETACICA DE ARGENTINA, BOLIVIA Y PERU

(F. C. Reyes) DIB.H.I.R.

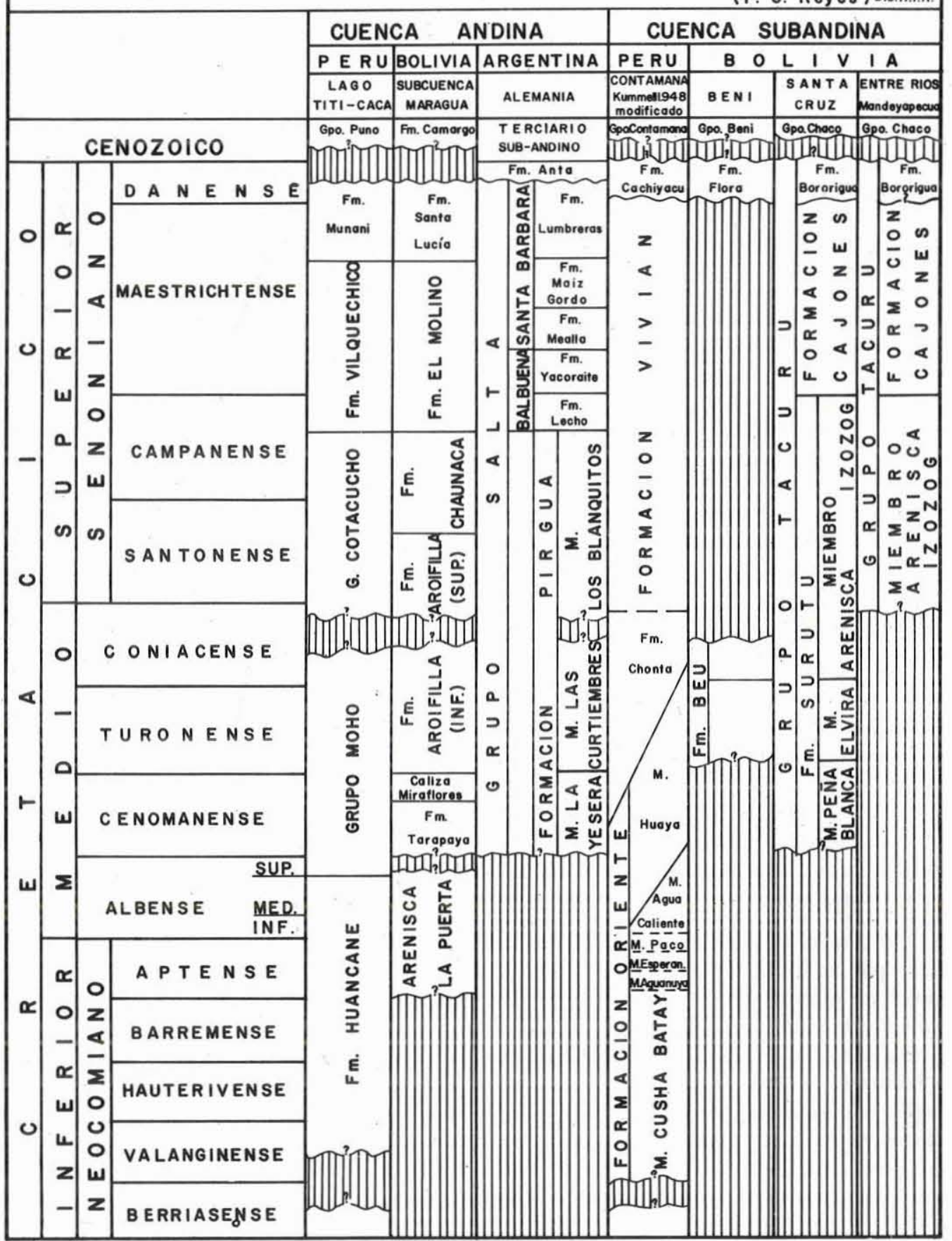


Yanamayu, cerca de la frontera Boliviana-Peruana, de pertenecer al mismo ciclo efusivo, parece indicar que el "Basalto de Entre Ríos" al menos en parte sería posterior a la deposición del Miembro Elvira (Formación Beu).

La secuencia cretácica de la Cuenca Subandina del Perí (Región de Contamana) La secuencia cretácica aflorante en la Región de Contamana (Perú), según Kummel (1948), es divisible en cuatro formaciones que sobrepasan los $2500 \mathrm{~m}$ de espesor. Ellas son, de abajo hacia arriba (Fig. 4):

1) Formación Oriente, con 1000 a $1700 \mathrm{~m}$ de potencia en la cual se recocen varios miembros. Esta formación está formada mayormente por areniscas de grano grueso, macizas, entrecruzadas y con algunas intercalaciones lutíticas. Una lengua, correspondiente al Miembro Esperanza, contiene fósiles marinos aptianos. Otra lengua marina en el Miembro Huaya proporcionó fósiles marinos del Albiano inferior y Albiano medio-inferior. Fuera de estas dos lenguas marinas y según lo manifestado por el mismo Kummel, el resto parece de origen continental.

2) Formación Chonta. Esta unidad litoestratigráfica, según Kummel (op. cit.), está compuesta, en la región de Contamana, de lutitas gris oscuras con intercalaciones de limolitas y limolitas calcáreas y algunas calizas. Hacia el Sur, sobre el Río Pachitea la Formación Chonta incluiría calizas macizas. El contacto con la Formación Oriente infrayacente, como con la Formación. Vivian que le suprayace, sería, según Kummel (op. cit.) concordante. El espesor en la Región de Contamana llega a $400 \mathrm{~m}$ en el Río Cushabatay (op. cit.), es una formación muy fosilífera. En la sección mencionada de Cushabatay, en su porción basal lleva fósiles de edad Turoniana (Inoceramus labiatus Schlotheim), mientras que la parte superior es Coniaciana, como acontece también en la sección de Pachitea.

3) Formación Vivian. Esta formación, según Kummel (op. cit.), es una arenisca entrecruzada de grano grueso a fino de color castaño-amarillento a blanquecino y con capas de lutitas negras interestratificadas. Los contactos superior e inferior se estipulan como aparentemente concordantes, aunque Kummel puntualiza que la mayoría de los contactos entre las Formaciones Vivian y Cachiyacu es de origen tectónico o probremente expuestos. A lo largo del Río Cushabatay su espesor alcanza los $220 \mathrm{~m}$ (Kummel, 1948). Ningún fósil diagnóstico se habría encontrado en la Formación Vivian fuera de restos de hojas de Monocotiledóneas y Dicotiledóneas no identificables. Sin embargo, Kummel la interpreta como una facies costera regresiva (p. 1253, op. cit.) del mar Coniaciano.

4) Formación Cachiyacu. Esta formación, según Kummel (op. cit.), está formada por una serie de lutitas negras, arcilitas margosas y limolitas conteniendo una fauna de agua salobre a marina. Suprayace a la Formación Vivian e infrayace al Grupo Contamana de red-beds de edad Terciaria, con aparente concordancia. En la región de las Montañas de Contamana, la Formación Cachiyacu tiene aproximadamente $150 \mathrm{~m}$ de potencia. Se ha encontrado en esta unidad numerosas ostras y dientes de tiburones no diagnósticos que sugieren condiciones marinas a salobres (Kummel, op. cit.). Los ostrácodos habrían sido identificados como Cypris y Metacypris. Se tratarían de sedimentitas depositadas durante la última transgresión registrada en la región de Contamana (Kummel, op. cit.). Este autor menciona igualmente que la pequeña colección de la Isla de Chonta, recogidos por Singewald y estudiados por Pilsbry, sería paralelizable con la Formación Cachiyacu, conteniendo ejemplares de Gyrodes que Pilsbry los halla muy relacionados con formas descritas para las capas Quiriquina de la Bahía Concepción, Chile, consideradas Maestrichtianas en base a amonites. 
Tentativa de correlación entre las unidades litoestratigráficas de la Cuenca Subandina de Bolivia con esas aflorantes en el Oriente del Perú y en la Cuenca Andina de Perú, Bolivia y Argentina Esta tentativa se halla esquematizada en la Fig. 4. Sintetizando las relaciones establecidas puede decirse:

1. En la Cuenca Subandina de Bolivia faltaría casi toda la secuencia Cretácica correspondiente a la Formación Oriente de la Región de Contamana, Perú y por lo tanto sus equivalentes laterales de la Cuenca Andina: Formaciones Huancané (Lago Titicaca) y La Puerta (Bolivia). Sólo podrían hallarse representados los niveles paralelizables del Miembro Huaya de la Formación Oriente en aquellas unidades basales de la Formación Surutú (Miembros Peña Blanca y Elvira). Sin embargo, esta última relación no puede ser probada por la carencia absoluta de fósiles en estas dos últimas unidades litoestratigráficas, aunque no puede descartarse del todo, de que al menos el Miembro Peña Blanca de la Formación Surutú pudiera ser aún más viejo y equivalente a la Formación Huancané.

De todas maneras dentro del Grupo Tacurú necesariamente se encuentran sedimentitas más viejas de lo que hasta ahora se habría sospechado.

Con el criterio conservador de la presente correlación (Fig. 4), se hallarían representadas en la parte basal del Grupo Tacurú sedimentitas del Cenomaniano y Turoniano, conocidas en la Guenca Andina del Perú y Bolivia (Grupo Moho y Formaciones Tarapaya, Miraflores y Aroifilla), paralelizables éstas en un otro y último trabajo del autor, con los Miembros La Yesera y Las Curtiembres dé la Subcuenca de Alemanía (Argentina) de la Formación Pirgua.

2. Las efusiones correspondientes al Basalto de Entre Ríos se podrían corresponder en tiempo con aquella segunda fase magmática efusiva que la hizo conocer Russo y Rodrigo Gainza (1965) para la Cuenca Andina de Bolivia en el área de Betanzos y cuya determinación radiactiva de su edad acusó 82,5 m.a., o sea, Coniaciana tardía (Kriz, Cherroni et al., 1966). Asimismo encontraría su correspondencia con las efusiones basálticas de la Subcuenca de Alemanía (Salta, Argentina), acaecidas durante los movimientos intersenonianos o peruanos. El autor se inclina a correlacionar la parte alta de la Formación Chonta que, por otra parte dado el carácter transgresivo de esta unidad, sería la que podría hallarse representada en territorio boliviano, con la parte basal e inferior del Miembro Arenisca Izozog de la Formación Surutú (Figs. 2 y 4).

La parte superior del Miembro Arenisca Izozog de la Cuenca Subandina de Bolivia, que contiene los primeros Pucalithus de la sección cretácica, podría correlacionarse con la parte inferior de la Formación Vivian del Oriente peruano y con la Formación Cotacucho y parte basal de Vilquechico de la región andina del Lago Titicaca. Asimismo podría corresponder con las Formaciones Aroifilla (superior), Formación Chaunaca y la parte basal de la Formación El Molino de la Cuenca Andina de Bolivia o bien con el Miembro Los Blanquitos de la Formación Pirgua y Formación Lecho de la Subcuenca de Alemania de Salta (Argentina).

3. La Formación Cajones de la Cuenca Subandina de Bolivia podría corresponderse con la parte superior de la Formación Vivian del Oriente peruano y con las Formaciones Vilquechico, El Molino, Muñani y Santa Lucía, de la Cuenca Andina de Perú y Bolivia y de los Subgrupos Balbuena y Santa Bárbara del Grupo Salta de Argentina.

4. La Formación Bororigua, que en la Subcuenca de Santa Cruz tiene un conglomerado de base, el autor, debido a las consideraciones expuestas en otra parte del presente trabajo, se inclina a correlacionarla con la Formación Flora de la Subcuenca Beniana 
de Bolivia y con la Formación Cachiyacu del Oriente Peruano. Asimismo cree que la Formación Anta de la Subcuenca de Alemanía y Metán, que se apoya con cierta discordancia sobre la Formación Lumbrera (Margas Coloradas Superiores), y que proporcionó fósiles cretácicos y el mismo Pucalithus, puede ser paralelizable con la Formación Flora.

\section{LISTA DE TRABAJOS CITADOS EN EL TEXTO}

BONARELLI, G. - 1921 - Tercera contribución al conocimiento geológico de las regiones petroliferas subandinas del Norte (Prov. de Salta y Jujuy). Buenos Aires, An. Min. Agric., Dir. Gral. Min., Geol. e Hidrol., XV (1)

CANEDO REYES, R. - 1960 - Informe sobre la geología de la zona petrolifera del Noroeste. La Paz, Bol. I. B. P., I (2): 9-31

GECIONI, G. - 1961 - El Titónico inferior marino en la Provincia de Tarapacá y consideraciones sobre el arqueamiento central de los Andes. Univ. Chile, Santiago de Chile, Com. Esc. Geol., $1(3)$

DIAZ, H. - 1959 - Comunicación acerca de las condiciones geológicas presentes en el curso superior del Río Beni. La Paz, Bol. Técn. Y. P. F. B., 1 (2): 21-27

HARRINGTON, G. L. - 1926 - Geologic report on the upper Rio Beni region Bolivia (informe inedito S. O. Co. of Bolivia)

HARRINGTON, H. J. - 1962 - Paleogeographic development of South America. Tulsa, Oklahoma, Bull. A. A. P. G., 46 (10): 1 773-1 814

HEALD, K. C. y MATHER, K. F. - 1922 - A reconnaissance of the eastern Andes between Cochabamba and Santa Cruz, Bolivia. New York, Bull. Geol. Soc. Am., 33: 553-570

KRIZ, S. J., CHERRONI, M. C. et al. - 1966 - Diagramas correlativos de formaciones cretácicas del Sudoeste de Bolivia. Serv. Geol. de Bolivia, La Paz

KUMMEL, B. - 1948 - Geological Reconnaissance of the Contamana Region, Peru. New York, Bull. Geol. Soc. Am., 59: 1 217-1 266

LAMB, W. G. y TRUITT, P. - 1963a - The Petroleum Geology of the Santa Cruz area Bolivia. Frankfurt \& Main, Sixth World Petr. Congr., Sec. I, paper 40, 573-594

LAMB, W. G. y TRUITT, P. - 1963b - Geología del Petróleo en el área de Santa Cruz. La Paz, Bol. I. B. P., 4 (1-IV): 12-39

LOPEZ MURILLO, H. - 1967 - Acerca de la geología de las Serranias Subandinas del Noroeste. La Paz, Bol. I. B. P., 7 (2): 14-27

MATHER, K. F. - 1922 - Front Ranges of the Andes between Santa Cruz, Bolivia and Embarcación, Argentina. New York, Bull. Geol. Soc. Am., 33: 703-764

MAURI, E. T., PADULA, E. L. y ALDAZOSA, J. - 1956 - Yacimientos de Petróleo y Gas en Bolivia. Simposium de Yac. de Petr. y Gas América del Sur y Antillas, t. IV, XX Congr. Geol. Int., México

OBLITAS, J. - 1962 - Informe geológico Anticlinal de Cuevo, Anticlinal Guairui Sur (sector meridional), Estructura de Bororigua (inf. inédito Y. P. F. B.), La Paz

PADULA, L. E. y MIANGRAMM, A. - 1968 - Estratigrafia, distribución y cuadro geotectónico-sedimentario del "Triásico" en el subsuelo de la Llanura Chaco-Paranense. Buenos Aires, Terc. Jorn. Geol. Arg., 1: 291-331

PADULA, L. E. y REYES, F. C. - 1958 - Contribución al Léxico estratigráfico de las Sierras Subandinas-República de Bolivia. La Paz, Bol. Técn. Y. P. F. B., I (1)

PERRY, L. D. - 1963 - Flora Formation (Upper Cretaceous of Northern Bolivia). Tulsa, A. A. P. G., $47(10): 1835-1860$

REYES, F. C. - 1955 - Informe geologico sobre la Serranía de Mandeyapecua-Carandaití, sector Ibamiranti-Nuapua, Prov. Azero, Dpto. Chuquisaca (inf, inéd, Y.P.F.B.), La Paz

REYES, F. C. - 1962 - - "Bolivia". Roma Encicl. Petrolio e del Gas Natural a cura dell' E. N. I., I: $787-806$

REYES, F. G. - 1963 - Correlaciones estratigráficas postcarboniferas (nivel de referencia: tope Carbonffero (Gondwana). Inéd. Y. P. F. B., La Paz 
REYES, F. G. y OBLITAS, J. - 1968 - Correlaciones estratigráficas postcarboníferas en la Cuenca. Subandina de Bolivia. Est. inéd. Y. P. F. B., La Paz

RUSSO, A. - 1955a - Informe geologico sobre la Serranía de Mandeyapecua-Carandaiti, sector Yoay-Ibamiranti (Inf. inéd. Y.P. F. B.), La Paz

RUSSO, A. - 1955b - Levantamiento geológico de las Serranías del Obispo y Terebinto, Dpto. Santa Cruz. Inf. inéd. Y. P. F. B., La Paz

RUSSO, A. y RODRIGO GAINZA, L. A. - 1965 - Estratigrafia y paleogeografia del Grupo Puca en Bolivia. La Paz, Bol. I. B. P., 5 (3-4): 5-5 l

SCHLAGINTWEIT, O. - 1939 - Informe preliminar sobre reconocimientos entre San Borja y Huachi y en el Río Beni entre Huachi y Rurrenabaque. Inf. inéd. Y. P. F. B., La Paz

SCHLATTER, L. E. y NEDERLOF, N. H. - 1966 - Bosquejo de la Geologia y paleogeografia de Bolivia. La Paz, Serv. Geol. Bolivia, Bol. 8

SUAREZ ROCA, H. - 1960 - Informe geológico área Angostura, Buena Vista, Anticlinales Caranda, Terebinto, Espejos y Cheyu, Prov. Ichilo e Ibañez, Dpto. Santa Cruz. Inf. inéd. Y.P. F. B., La Paz

WILSON, J. J. - 1963 - Cretaceous Stratigraphy of Central Andes of Peru. Tulsa, Bull. A. A. P. G., 47 (1): 1-34

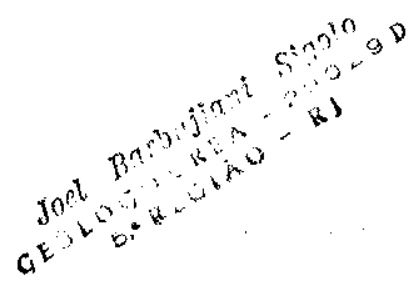

